sis. Indeed this is borne out by the prevalence figure for the Western Isles of Scotland ${ }^{2}$ of only 81 per 100000 , which is one of the lowest prevalence rates found in the United Kingdom and yet these islands have the highest percentage of Scottish surnames.

DAVID I SHEPHERD Department of Neurology, North Manchester General Hospital, Crumpsall, Manchester M8 6RB, UK

1 Rothwell PM, Charlton D. High incidence and prevalence of multiple sclerosis in south east prevalence of multiple sclerosis in south east F Neun. Neurosurg Pychiatry 1998;64:730-5. f Neurol Neurosurg Psychiatry 1998;64:730-5.

2 Dean G, Downie A, Goodall J. multiple sclerosis in the Outer Hebrides. In: Clifford Rose F, ed. Clinical neuroepidemiology. Tunbridge Wells: Pitman medical, 1980:219-21.

High incidence and prevalence of multiple sclerosis in south east Scotland: evidence of a genetic predisposition

We read with interest the results of Rothwell and Charlton regarding the incidence and prevalence of multiple sclerosis in south east Scotland. ${ }^{1}$ They have identified standardised multiple sclerosis prevalence rates for the Lothian and Border Regions of 203 and 219 per 100000 respectively, the results challenging the theory that the high prevalence rates previously reported in Scotland are peculiar to the north east and its offshore islands. The authors postulate that the apparent step in prevalence rates between England and Scotland may be due to the distinctive Celtic ancestry of the Scottish population as can be crudely measured by surnames prefixed with Mc or Mac.

In Northern Ireland we have also identified a much higher prevalence rate for the disease than exists in England and Wales and have speculated that the similar rate to that in Scotland is at leastly partly a function of the common ethnic origins of the two populations. ${ }^{2}$ The contiguous region of Coleraine, Moyle, Ballymena, and Ballymoney lies less than 20 miles from Scotland at its closest point and has a standardised prevalence rate for all multiple sclerosis, based on the 1961 census population for Northern Ireland, ${ }^{3}$ of 230 (95\% confidence interval (95\% Cl) 207-256) per 100 000. Using a similar method to Rothwell and Charlton (British Telecom phone book of the area), $17 \%$ of the study population had a surname prefixed with Mc or Mac and it is of note that $22.9 \%$ of prevalent cases had such a surname prefix (odds ratio $=1.46,95 \% \mathrm{Cl} 1.09-1.93$, $\left.\chi^{2}=6.82, p=0.009\right)$.

Our results support the conclusion of Rothwell and Charlton that Celtic ancestry is a risk factor for multiple sclerosis and confirm the existence of a step in multiple sclerosis prevalence in the British Isles between England/Wales and Scotland/ Northern Ireland.

G V MCDONNELL S A HAWKINS Northern Ireland Neurology Service, Quin House, Royal Victoria Hospital, Grosvenor Road, Belfast, Northern Ireland BT12 6BA, UK

1 Rothwell PM, Charlton D. High incidence and prevalence of multiple sclerosis in south east Scotland: evidence of a genetic predisposition.

2 McDonnell GV, Hawkins SA. An epidemiologic McDonnell GV, Hawkins SA. An epidemiologic
study of multiple sclerosis in Northern Ireland. study of multiple sclerosis in
Neurology 1998;50:423-8.
3 Millar JHD. Multiple sclerosis in Northern Ireland. In: Clifford Rose F, ed. Clinical neuroepidemiology. Tunbridge Wells: Pitman Medical, 1980:222-7.

The author's reply:

The study of the prevalence of multiple sclerosis in Northern Ireland by McDonnell and Hawkins is interesting. ${ }^{1}$ The findings are similar to those of the recent study in south east Scotland. ${ }^{2}$ Both studies suggest that there is an increased prevalence of multiple sclerosis in the north of the British Isles compared with the south. It seems likely, as McDonnell and Hawkins suggest, that this at least partly reflects differences in the genetic susceptibility of the respective populations.

The south east Scotland study did, as Shepherd suggests, attempt to link the high prevalence of multiple sclerosis to Scottish ancestry. However, the study used a standard text of several hundred surnames which are considered to have originated in Scotland, ${ }^{3}$ rather than just those prefixed with Mc or Mac. This is obviously still a very crude approach to the problem, but any bias is likely to have weakened rather than strengthened the association. The proportion of cases in the telephone book with a surname pre-fixed with Mc or Mac was simply used as a crude illustration of the fact that the differences in apparent ancestry between the Scotland and England are still considerable. This is supported by major differences in the HLA types of the two populations. ${ }^{4}$ Contrary to Shepherd's assertion, the Highlands and Islands telephone book does include Orkney. However, he is correct to point out that the prevalence of surnames pre-fixed with $\mathrm{Mc}$ or Mac is indeed lower on Orkney than in the region as a whole.

Further insights into the high prevalence of multiple sclerosis in the north of the British Isles might come from a prevalence study which is currently being planned on the Isle of Skye.

P M ROTHWELL

Department of Clinical Neurology, Radcliffe Infirmary, Woodstock Road, Oxford OX2 6HE, UK

1 McDonnell GV, Hawkins SA. An epidemiologic study of multiple sclerosis in Northern Ireland. study of multiple sclerosis
Neurology 1988;50:423-8.

2 Rothwell PM, Charlton D. High incidence and prevalence of multiple sclerosis in south east Scotland: evidence of a genetic predisposition. f Neurol Neurosurg Psychiatry 1998;64:730-5.

3 Guppy HB. Homes of family names in Great Britain. Harrison, 1980

4 Swingler RJ, Compston DAS. The distribution of multiple sclerosis in the United Kingdom. $\mathcal{F}$ Neurol Neurosurg Psychiatry 1986;49:1115-24.

\section{Albendazole therapy for subarachnoid cysticerci: clinical and neuroimaging analysis of 17 patients}

By contrast with the weaknesses of anecdotal observations from case series, the power of randomised clinical trials for deciding the benefit of therapy has become increasingly evident and indisputable world wide. Nowadays, to argue against the validity of this assertion may seem superfluous; however, a recent paper reported by Del Brutto ${ }^{1}$ regarding treatment in neurocysticercosis ignores basic procedures for well performed clinical trials by using inappropriate and misleading methodology to evaluate medical therapy.

By definition, a clinical trial is a prospective study comparing the effect and value of treat- ment against a control in human subjects. The main drawback of Del Brutto's report is that it does not include a control group against which the intervention group is compared; therefore, its results are definitely flawed. Additionally, a basic experimental study design requires at least minimal information regarding inclusion and exclusion criteria, randomisation, and definitions of response or outcome variables. This information is not provided by Del Brutto's report; its design fails to protect against potential bias in patient selection or evaluation of outcome. The definition of subarachnoid cysterci used by Del Brutto was based on "appearance on CT of hypodense cystic lesions located over the convexity of the cerebral hemispheres, the sylvian fissure, or the CSF cisterns at the base of the brain". It is well known that there are many other diagnostic possibilities to be considered in the differential diagnosis of subarachnoid hypodense lesions. ${ }^{23}$ Besides, CT is not a reliable procedure for diagnosing subarachnoid cysterci, as is MRI. In fact, we cannot be completely sure, for example, that the CT images shown in the report of Del Brutto correspond to subarachnoid cysterci. If we were to use MRI on this patient, they might correspond to a parenchymal cyst which resolved as a reflection of the natural history of the condition. There is no evidence that objectively confirms or rejects this assertion.

Del Brutto's report ${ }^{1}$ maintains that evaluation of the therapeutic response to albendazole included comparison of the size of the cysts, as well as clinical evaluation of patients before and after treatment. To consider the size of cysts as a response variable is certainly useless because of the obvious difficulties in measuring cyst size in the subsequent follow up CT. It is also widely accepted that the clinical manifestations of neurocysticercosis are polymorphic, and their clinical course is unpredictable; therefore, the clinical manifestations as an outcome variable is entirely biased. Another personal appreciation of Del Brutto $^{1}$ is that albendazole reaches high concentrations in CSF, and has been used with success in some patients with subarachnoid cysts; nevertheless, studies used as support ${ }^{1}$ of this presumption are similarly flawed in that they are not randomised or blinded, having historical control groups or patients who served as their own control, and regarding clinical evaluation as an outcome variable.

Whereas it is generally assumed that albendazole is effective treatment for neurocysticercosis, a critical review of the literature ${ }^{34}$ suggests that the studies on which these assumptions are based are defective in terms of patient selection, assignment to treatment, and selection and measurement of outcome variables. Many authors have warned that this therapy in some patients might sometimes be harmful, particularly in the subarachnoideal localisation, because some patients have developed arteritis and hydrocephalus after the administration of antihelminthic drugs. ${ }^{3}$ According to these authors a parasite may be easily removed surgically at a cystic stage before an inflammatory reaction develops. ${ }^{3} \mathrm{~A}$ randomised clinical trial of treatment of neurocysticercosis ${ }^{4}$ considers the question of to what extent and in which patients treatment with either praziquantel or albendazole is effective. The improvement attributed to these drugs in several studies may be related to the lack of appropriate controls and is likely to be a reflection of the natural history of the condition. The authors point 
out the need to conduct a long term, placebo controlled trial with precise end points, proper randomisation, sample size calculations, and predetermined statistical calculations, to evaluate properly the effectiveness and determine the indications of aetiological treatment for neurocysticercosis.

In the era of evidence-based medicine, we neurologists and general practitioners should be demanding regarding use of sound scientific information with methodological rigour for improving our clinical decision making. ${ }^{5}$ Medical information from reports that do not conform to the minimal requirements of a clinical trial should be avoided.

A CARPIO

University of Cuenca School of Medicine, PO Box 0101-719, Cuenca, Ecuador Correspondence to: Professor A Carpio, University of Cuenca School of Medicine, PO Box 0101-719, Cuenca, Ecuador.

\section{Del Brutto OH. Albendazole therapy for subarachnoid cysticerci: clinical and neuroim- aging analysis of 17 patients. $\mathcal{F}$ Neurol Neurosur Psychiatry 1997;62:659-61 \\ 2 Carpio A, Placencia M, Santillan F, et al. Proposal for a new classification of neurocyst- icercosis. Can F Neurol Sci 1994;21:43-7. \\ 3 Caplan LR, Estanol B, Mitchel WG, et al. How to manage patients with neurocysticercosis. Eur Neurol 1997;37:124-31. \\ 4 Carpio A, Santillan F, Leon P, et al. Is the course of neurocysticercosis modified by treatmen with antihelminthic agents? Arch Intern Med 1995;155:1982-8. \\ 5 Sackett DL, Rosenberg WMC, Gray JAM, et al. Evidence based medicine: what it is and what it isn't. It's about integrating individual clinical isn't. It's about integrating individual clinical
expertise and the best external evidence. $B M Y$ expertise and the}

The author's reply:

I celebrate the rigid academic standards of Carpio's medical practice, but wish they were matched with knowledge on the available literature on albendazole therapy for neurocysticercosis. That albendazole actually has a cysticidal effect is beyond all doubt. ${ }^{1-6}$ The drug has been used to treat patients with neurocysticercosis since 1987 , and physicians who are familiar with the disease know that it is effective. Moreover, the single study in which albendazole has not been useful for adults with viable parenchymal brain cysticerci-published by Carpio et al-has been questioned due to inaccurate recollection of data. ${ }^{8}$ In our study, we did not attempt to verify the cysticidal effect of the drug (it has already been demonstrated) but to document if albendazole could also be useful in a severe form of neurocysticercosis that has been associated with a grim prognosis.' Under these circumstances, it is not ethical to deprive a group of patients of a safe and inexpensive treatment just for the sake of science. In addition, Carpio's concerns about the criteria we used for the diagnosis of subarachnoid cysticerci are typical of those who are not familiar with the disease. The problem with $\mathrm{CT}$ is that this imaging method may misdiagnose some subarachnoid cysts as parenchymal cysts, but the opposite is not true, ${ }^{10}$

As a physician interested in the advancement of science, I applaud Carpio's interest in evidence-based medicine but I completely disagree with him in that information from reports other than clinical trials should be avoided. He must remember that outstanding contributions to medical knowledge have been made through single case reports, small clinical series, and open trials. On the contrary, the cliché "randomised" has been the shield of major medical frauds. ${ }^{11}$ Medicine is art and science, and wise physicians know that information from clinical findings actually have a "significant" impact on everyday clinical practice.

OSCAR H DEL BRUTTO

Department of Neurology, Luis Vernaza Hospital, Guayaquil, Ecuador

1 Sotelo J, Penagos P, Escobedo F, et al. Short course of albendazole therapy for neurocysticercosis. Arch Neurol 1988;45:1130-3.

2 Cruz I, Cruz M, Carrasco F, et al. Neurocysticercosis: optimal dose treatment with albendazole. F Neurol Sci 1995;133:152-4.

3 Wakalondazole. Neurol Sci E. Th, Takayanagui OM, Jardim E. Therapy for neurocysticercosis: comparison between albendazole and

4 García HH, Gilman RH, Horton J, et al. Albendazole therapy for neurocysticercosis: a prospective double-blind trial comparing 7 versus 14 days of treatment. Neurology 1997;48:14217

5 Alarcón F, Escalante L, Dueñas G, et al. Neurocysticercosis: short course of treatmen with albendazole. Arch Neurol 1989;46:1231-6.

6 Del Brutto OH. Medical treatment of cysticercosis-Effective. Arch Neurol 1995;52: 102-4.

7 Carpio A, Santillán F, León P, et al. Is the course of neurocysticercosis modified by treatment with antihelminthic ants? Arch Intern Med 1995;155:1982-8.

8 Del Brutto OH. Is the course of neurocysticercosis modified by treatment with antihelmincosis modified by treatment with antihelmin-
thic agents [letter]? Arch Intern Med 1997;157: thic age

9 Del Brutto OH. Albendazole therapy for Del Brutto OH. Albendazole therapy for
subarachnoid cysticerci: clinical and neuroimaging analysis of 17 patients. F Neurol Neurosurg Psychiatry 1997;62:659-61.

10 Del Brutto OH, Sotelo J, Román G. Neurocysticercosis: a clinical handbook. Lisse, The Netherlands: Swets and Zeitlinger, 1998.

11 Woolf PK. Ensuring integrity in biomedical publication. $\mathcal{F A M A}$ 1987;258:3424-7.

\section{BOOK REVIEWS}

Stroke Units: An Evidence Based Approach. Edited by PETER LANGHORNe and MARTIN DenNis. (Pp112, £25.00). Published by BMJ Books, London 1998. ISBN 0-7279-1211-9.

Ever since the landmark overview "Do stroke units save lives?" the momentum behind the organisation of stroke sevices has inexorably increased. Within this concise paperback of seven chapters and 112 pages, written in the main by Langhorne and Dennis on behalf of the Stroke Unit Trialists Collaboration, lies a detailed, evidence-based, and critical summary of the arguments for stroke units.

The authors lead the reader in a logical fashion through the steps necessary to critically appraise and assimilate available evidence into a systematic review. Basic but often overlooked general principles such as sources of bias are discussed in detail as well as matters relating more specifically to stroke such as outcome measurement.

Particularly helpful are the chapters on the economics of stroke units and the implications for service planning in which the available evidence has been used to suggest how and at what cost (in fact an overall saving) stroke units might be developed.

This comprehensively referenced book will be read by members of all disciplines involved in stroke care. If I was about to ask for fund- ing for a stroke unit I would certainly have it in my pocket!

PETER MARTIN

Immunological and Infectious Diseases of the Peripheral Nerves. Edited by NORMAN LATOV, JOHN H J WOKKE, and JOHN J KELLY. ( $\$ 95.00, \quad$ US $\$ 145.00$ (HB)). Published by Cambridge University Press, Cambridge 1998. ISBN 0-521-46265-7.

There are two parallel strands to the development of our understanding of immune mediated disorders of peripheral nerve. The first grew from the demonstration in the 1950s, by Waksman and Adams, that rabbits immunised with homologous sciatic nerve and adjuvant developed an inflammatory demyelinating neuropathy. In this model, experimental allergic neuritis, the CSF characteristically shows a raised protein concentration and a paucity of cells. These findings replicated those of Guillain-Barré and Strohl on the CSF abnormalities of Landry's disease and so spawned the notion that GuillainBarré syndrome was immunologically mediated. Accordingly, over the past 20 years patients with Guillain-Barré syndrome and chronic inflammatory demyelinating peripheral neuropathy have been exposed to immunosuppressive regimes borrowed from other inflammatory disorders.

The second important development has been the growing understanding of the relation between plasma cell dyscrasias and peripheral neuropathies. The association of peripheral neuropathy and myeloma was noted in the 1930s and that with an IgM monoclonal gammopathy was reported in the 1960s. Twenty years later IgM antibodies were found that were directed against myelin associated glycoprotein. This work has gathered pace and over the past 10 years, peripheral neuropathies have been described in association with specific antiganglioside and antisulphatide antibodies.

Alongside this expansion of interest in the immunology of peripheral nerve disorders, new infective neuropathies have emerged such as those due to HIV and Lyme disease, first recognised in 1993. It is an appropriate time then for this authoritative text on immune mediated neuropathies. The scope of the book is wide, including scientific overviews of immune interactions in the peripheral nervous system as well as pragmatic accounts of the use of immunosuppressant drugs and the management of neuropathic pain. The inflammatory demyelinating neuropathies and antibody associated neuropathies are comprehensively surveyed, as well as more difficult entities such as the post-polio syndrome and the rare toxic inflammatory neuropathies. The dry review of silicone neurotoxicity by Rosenberg is a special treat. British readers may be surprised to find only one United Kingdom contributor to this American-Dutch edited text, whereas 18 authors are American, 11 are from The Netherlands, two each from Italy, Japan, and Israel, and one each from Canada, Nepal, and Switzerland. It is not cheap, but it has no equal as a comprehensive, accessible, and useful resource for the practising neurologist.

ALASDAIR COLES 